\title{
Phototoxicity Secondary to Home Fireplace Exposure After Photodynamic Therapy for Actinic Keratosis
}

\author{
Lauren A. Hassoun, MD, MAS; Vivian Y. Shi, MD; Raja K. Sivamani, MD, MS, AP
}

\section{PRACTICE POINTS}

- As the applications of photodynamic therapy (PDT) in dermatology continue to expand, it is imperative for providers and patients alike to be knowledgeable with aftercare instructions and potential adverse effects.

- Avoid open flames in the house or while camping following PDT to maximize patient safety and prevent phototoxicity.

To the Editor:

Photodynamic therapy (PDT) is a US Food and Drug Administration-approved treatment for actinic keratosis (AK). It also commonly is administered off label for basal cell carcinoma, Bowen disease, photoaging, and acne vulgaris and is being investigated for other applications. ${ }^{1,2}$ In the context of treating $\mathrm{AK}$, the mechanism employed in PDT most commonly involves the application of exogenous aminolevulinic acid (ALA), which is metabolized to the endogenous photosensitizer protoporphyrin IX
(PpIX) in skin cells by enzymes in the heme biosynthetic pathway. ${ }^{3}$ The preferential uptake of ALA and conversion to PpIX is due to the altered and increased permeability of abnormal keratin layers of aging, sun-damaged cells, and skin tumors. Selectivity of ALA also occurs due to the preferential intracellular accumulation of PpIX in proliferating, relatively iron-deficient, precancerous and cancerous cells. The therapeutic effect is achieved with light exposure to blue light wavelength at $417 \mathrm{~nm}$ and corresponds to the excitation peak of $\mathrm{PpIX},{ }^{4}$ which activates PpIX and forms reactive oxygen species in the presence of oxygen that ultimately cause cell necrosis and apoptosis. ${ }^{5}$ Because it takes approximately 24 hours for PpIX to be completely metabolized from the skin, patients are counseled to avoid sun or artificial light exposure in the first 24 hours post-PDT, regardless of the indication, to avoid a severe phototoxic reaction. ${ }^{3,6,7}$ Although it is normal and desirable for patients to experience some form of a phototoxic reaction, which may include erythema, edema, crusting, vesiculation, or erosion in most patients, these types of reactions most often are secondary to the intended exposure and incidental natural or artificial light

Dr. Hassoun is from the Department of Medicine, Baylor Scott and White Health System, Buda, Texas. Dr. Shi is from the Department of Dermatology, University of Arkansas for Medical Sciences, Little Rock. Dr. Sivamani is from the Department of Biological Sciences, California State University, Sacramento; Department of Dermatology, University of California Davis, Sacramento; Zen Dermatology, Sacramento; College of Medicine, California Northstate University, Elk Grove; and Pacific Skin Institute, Sacramento.

Dr. Hassoun reports no conflict of interest. Dr. Shi is on the board of directors for the Hidradenitis Suppurativa Foundation; is a stock shareholder of Learn Health; and has served as an advisory board member, investigator, speaker, and/or received research funding from AbbVie, Altus Lab/ cQuell, Aristea Therapeutics, Boehringer Ingelheim, Burt's Bees, Dermira, Eli Lilly and Company, Galderma, GpSkin, Incyte, Kiniksa, LEO Pharma, Menlo Therapeutics, MYOR, Novartis, Pfizer, Polyfins Techology, Regeneron, Sanofi Genzyme, Skin Actives Scientific, SUN Pharma, TargetPharmaSolutions, and UCB. Dr. Sivamani has served as an advisory board member and speaker for and has received funding and/or honoraria from AbbVie, Burt's Bees, Codex Beauty, Eli Lilly and Company, Incyte, LEO Pharma, Novozymes, Nutrafol, Regeneron, Sun Pharma, and UCB. Correspondence: Raja K. Sivamani, MD, MS, AP (raja.sivamani.md@gmail.com).

doi:10.12788/cutis.0392 
exposures. ${ }^{6}$ We report a case of a severe phototoxic reaction in which a patient experienced painful erythema and purulence on the left side of the chin after being within an arm's length of a flame in a fireplace following PDT treatment.

A 59-year-old man presented to our dermatology clinic for his second of 3 PDT sessions to treat AKs on the face. He had a history of a basal cell carcinoma on the left nasolabial fold that previously was treated with Mohs micrographic surgery and melanoma on the left ear that was previously treated with excision. The patient received the initial PDT session 1 month prior and experienced a mild reaction with minimal redness and peeling that resolved in 4 to 5 days. For the second treatment, per standard protocol at our clinic, ALA was applied to the face, after which the patient incubated for 1 hour prior to blue light exposure (mean [SD] peak output of 417 [5] nm for 1000 seconds and $10 \mathrm{~J} / \mathrm{cm}^{2}$ ).

After blue light exposure, broad-spectrum sunscreen (sun protection factor 47) was applied to our patient's face, and he wore a wide-brimmed hat upon leaving the clinic and walking to his car. Similar to the first PDT session 1 month prior, he experienced minimal pain immediately after treatment. Once home and approximately 4 to 5 hours after PDT, he tended to a fire using his left hand and leaned into the fireplace with the left side of his face, which was within an arm's length of the flames. Although his skin did not come in direct contact with the flames, the brief 2 - to 3 -minute exposure to the flame's light and heat produced an immediate intense burning pain that the patient likened to the pain of blue light exposure. Within 24 hours, he developed a severe inflammatory reaction that included erythema, edema, desquamation, and pustules on the left side of the chin and cheek that produced a purulent discharge (Figure). The purulence resolved the next day; however, the other clinical manifestations persisted for 1 week. Despite the discomfort and symptoms, our patient did not seek medical attention and instead managed his symptoms conservatively with cold compresses. Although he noticed an overall subjective improvement in the appearance of his face after this second treatment, he received a third treatment with PDT approximately 1 month later, which resulted in a response that was similar to his first visit.

Photodynamic therapy is an increasingly accepted treatment modality for a plethora of benign and malignant dermatologic conditions. Although blue and red light are the most common light sources utilized with PDT because their wavelengths (404-420 $\mathrm{nm}$ and $635 \mathrm{~nm}$, respectively) correspond to the excitation peaks of photosensitizers, alternative light sources increasingly are being explored. There is increasing interest in utilizing infrared (IR) light sources (700-1,000,000 nm) to penetrate deeper into the skin in the treatment of precancerous and cancerous lesions. Exposure to IR radiation is known to raise skin temperature via insideout dermal water absorption and is thought to be useful in PDT-ALA by promoting ALA penetration and its

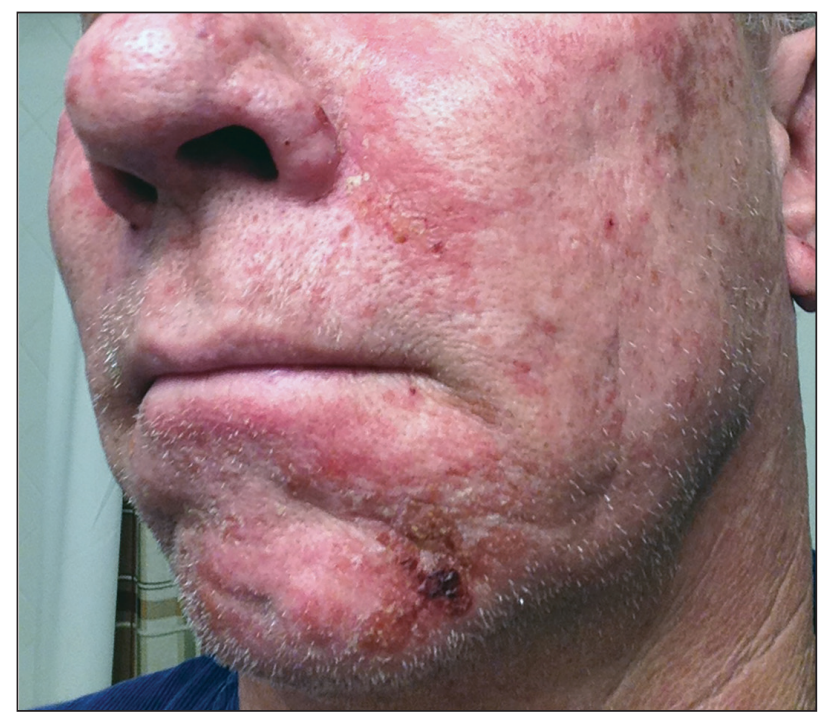

A severe phototoxic reaction developed within 24 hours of photodynamic therapy for actinic keratosis of the face after exposure to open flames in a fireplace. Erythema, edema, and purulent discharge were noted primarily on the left side of the chin.

conversion to $\mathrm{PpIX} .{ }^{8}$ In a randomized controlled trial by Giehl et al, ${ }^{9}$ visible light plus water-filtered IR-A light was shown to produce considerably less pain in ALA-PDT compared to placebo, though efficacy was not statistically affected. There are burgeoning trials examining the role of IR in treating dermatologic conditions such as acne, but research is still needed on ALA-PDT activated by IR radiation to target AKs.

Although the PDT side-effect profile of phototoxicity, dyspigmentation, and hypersensitivity is well documented, phototoxicity secondary to flame exposure is rare. In our patient, the synergistic effect of light and heat produced an exuberant phototoxic reaction. As the applications for PDT continue to broaden, this case may represent the importance of addressing additional precautions, such as avoiding open flames in the house or while camping, in the PDT aftercare instructions to maximize patient safety.

\section{REFERENCES}

1. Fritsch C, Ruzicka T. Fluorescence diagnosis and photodynamic therapy in dermatology from experimental state to clinic standard methods. J Environ Pathol Toxicol Oncol. 2006;25:425-439.

2. Lang K, Schulte KW, Ruzicka T, et al. Aminolevulinic acid (Levulan) in photodynamic therapy of actinic keratoses. Skin Therapy Lett. $2001 ; 6: 1-2,5$

3. Kennedy JC, Pottier RH. Endogenous protoporphyrin IX, a clinically useful photosensitizer for photodynamic therapy. J Photochem Photobiol B. 1992;14:275-292.

4. Wan MT, Lin JY. Current evidence and applications of photodynamic therapy in dermatology. Clin Cosmet Investig Dermatol. 2014;7:145-163.

5. Gad F, Viau G, Boushira M, et al. Photodynamic therapy with 5 -aminolevulinic acid induces apoptosis and caspase activation in malignant T cells. J Cutan Med Surg. 2001;5:8-13.

6. Piacquadio DJ, Chen DM, Farber HF, et al. Photodynamic therapy with aminolevulinic acid topical solution and visible blue light in the 
treatment of multiple actinic keratoses of the face and scalp: investigatorblinded, phase 3, multicenter trials. Arch Dermatol. 2004;140:41-46.

7. Rhodes LE, Tsoukas MM, Anderson RR, et al. Iontophoretic delivery of ALA provides a quantitative model for ALA pharmacokinetics and PpIX phototoxicity in human skin. J Invest Dermatol. 1997;108:87-91.
8. Dover JS, Phillips TJ, Arndt KA. Cutaneous effects and therapeutic uses of heat with emphasis on infrared radiation. J Am Acad Dermatol. 1989;20(2, pt 1):278-286.

9. Giehl KA, Kriz M, Grahovac M, et al. A controlled trial of photodynamic therapy of actinic keratosis comparing different red light sources. Eur J Dermatol. 2014;24:335-341. 